\title{
Erratum to: Quantum Mechanical Simulation of Cross Effect DNP Using Krylov-Bogolyubov Averaging
}

\author{
Alexander Karabanov • Grzegorz Kwiatkowski • \\ Walter Köckenberger
}

Published online: 28 August 2012

(C) Springer-Verlag 2012

\section{Erratum to: Appl Magn Reson (2012) 43:43-58 DOI 10.1007/s00723-012-0367-0}

Due to a processing error, the following References are incorrect:

References 7, 8, 11, 13-15, 17, 18 and 21.

The correct References are given below:

7. C.D. Jeffries, Phys. Rev. 117, 1056 (1960)

8. C.F. Hwang, D.A. Hill, Phys. Rev. Lett. 18, 110-112 (1967)

11. K.-N. Hu, H. Yu, T. Swager, R.G. Griffin, J. Am. Chem. Soc. 126, 10844-10845 (2004)

13. C. Song, K.-N. Hu, C.-G. Joo, T.M. Swager, R.G. Griffin, J. Am. Chem. Soc. 128, 11385-11390 (2006)

14. C. Ysacco, E. Rizzato, M.-A. Virolleaud, H. Karoui, A. Rockenbauer, F. Le Moigne, D. Siri, O. Ouari, R.G. Griffin, P. Tordo, Phys. Chem. Chem. Phys. 12, 5841-5845 (2010)

15. Y. Hovav, A. Feintuch, S. Vega, J. Magn. Reson. 214, 29-41 (2012)

17. N.M. Krylov, N.N. Bogoliubov, Introduction to Nonlinear Mechanics. (Princeton University Press, Princeton, 1947)

18. N.N. Bogoliubov, Y.A. Mitropolsky, Asymptotic Methods in the Theory of NonLinear Oscillations. (Hindustan Publishing Corporation, Dehli, 1961)

21. M.H. Levitt, L. Di Bari, Phys. Rev. Lett. 69, 3124-3127 (1992)

The online version of the original article can be found under doi:10.1007/s00723-012-0367-0.

\section{A. Karabanov · G. Kwiatkowski · W. Köckenberger $(\bowtie)$}

Sir Peter Mansfield Magnetic Resonance Centre, School of Physics and Astronomy,

University of Nottingham, Nottingham, UK

e-mail: walter.kockenberger@nottingham.ac.uk

A. Karabanov

e-mail: karabanov@hotmail.co.uk 